Department of Applied Mathematics

Faculty of EEMCS

University of Twente

The Netherlands
P.O. Box 217

7500 AE Enschede

The Netherlands

Phone: +31-53-4893400

Fax: +31-53-4893114

Email: memo@math.utwente.nl

www.math.utwente.nl/publications

Memorandum No. 1782

Pseudo-time stepping methods for

space-time discontinuous Galerkin

discretizations of the compressible

Navier-Stokes equations

C.M. Klaij, J.J.W. van DeR Vegt and

H. VAN DER VEN ${ }^{1}$

November, 2005

ISSN 0169-2690

${ }^{1}$ National Aerospace Laboratory NLR, P.O. Box 90502, 1006 BM Amsterdam, The Netherlands 


\title{
Pseudo-time stepping methods for space-time discontinuous Galerkin discretizations of the compressible Navier-Stokes equations
}

\author{
C.M. Klaij ${ }^{\mathrm{a}, *}$, J.J.W. van der Vegt ${ }^{\mathrm{a}}$, H. van der Ven ${ }^{\mathrm{b}}$ \\ ${ }^{a}$ University of Twente, Department of Applied Mathematics, \\ P.O. Box 217, 7500 AE Enschede, The Netherlands \\ ${ }^{\mathrm{b}}$ National Aerospace Laboratory NLR, \\ P.O. Box 90502, 1006 BM Amsterdam, The Netherlands
}

\begin{abstract}
The space-time discontinuous Galerkin discretization of the compressible NavierStokes equations results in a non-linear system of algebraic equations, which we solve with a local pseudo-time stepping method. Explicit Runge-Kutta methods developed for the Euler equations are unsuitable for this purpose as a severe stability constraint linked to the viscous part of the equations must be satisfied in boundary layers. In this paper, we investigate two new alternatives:
\end{abstract}

(1) an implicit-explicit Runge-Kutta method, where the viscous terms are treated implicitly and the inviscid terms explicitly,

(2) a combination of two explicit Runge-Kutta schemes, one designed for inviscid flows and the other for viscous flows.

We analyze the stability of the explicit and implicit-explicit methods, discuss their (dis)advantages and compare their performance by computing the flow around the NACA0012 airfoil at low and moderate Reynolds numbers.

Key words: compressible Navier-Stokes equations, discontinuous Galerkin finite element methods, pseudo-time integration, Runge-Kutta methods PACS: 02.60.Cb, 02.70.Dh, 03.40.Gc

* Corresponding author

Email addresses: c.m.klaij@math.utwente.nl (C.M. Klaij),

j.j.w.vandervegt@math. utwente.nl (J.J.W. van der Vegt), venvd@nlr.nl (H. van der Ven). 


\section{Introduction}

Discontinuous Galerkin (DG) methods are nowadays applied to a wide range of problems because of their accuracy and favorable properties related to the locality of the discretization, such as flexibility in mesh adaptation and efficient parallelization. In fact, the discretization in each element only involves its direct neighbors, even for higher order of accuracy, making the method most local. The usual approach is to apply discontinuous basis-functions in space and a Runge-Kutta method for the time integration, resulting in the so-called RKDG method, see for example the survey by Cockburn and Shu [9]. Thanks to the work by Bassi and Rebay [3], Baumann and Oden [5] and Cockburn and Shu [8], DG methods were successfully extended from hyperbolic to (incompletely) parabolic equations, see Arnold, Brezzi e.a. $[1,6]$ for the detailed analysis of purely elliptic problems and $[2,4,10]$ for applications to the compressible Navier-Stokes equations.

In [12], we presented a space-time discontinuous Galerkin method for the compressible Navier-Stokes equations, which is an extension of the space-time DG method for the Euler equations [18,19] designed for flow problems on moving and deforming meshes. The main idea is to use discontinuous basis-functions both in space and time, and to introduce a numerical time-flux to ensure causality in time. The viscous flux is treated by extending the approach presented in $[3,4]$ to the space-time context. The method is fully implicit in physical time and results in a system of non-linear algebraic equations [12]. This paper focuses on solving the algebraic system.

For the space-time discretization of the Euler equations [18], the algebraic system was solved with an explicit pseudo-time stepping Runge-Kutta method (with the correction by Melson e.a. [15]). When applied as a smoother in a full approximation multigrid scheme, this approach proved very efficient. The main advantage of the pseudo-time stepping method is its locality, which matches the locality of the discontinuous Galerkin method. An alternative would be to solve the system with a Newton method; in which case a global linear system based on the expensive Jacobian of the Euler flux would have to be solved. Another disadvantage of Newton's method is the small basin of attraction which demands an accurate initial guess in order to converge. In practice, this translates to small physical times steps for the space-time discontinuous Galerkin method. Being implicit in physical time, the solver of the algebraic system needs to be stable for large physical time steps as well. This is the case for the pseudo-time stepping method as it is insensitive to the initial condition. Therefore, we will aim at extending the pseudo-time stepping approach in [18] to the space-time discretization of the Navier-Stokes equations presented in $[12]$. 
Such an extension is not trivial for two reasons. First, for the Euler equations, the pseudo-time Courant-Friedrichs-Levy (CFL) condition must be satisfied for stability of the Runge-Kutta method [18]. But applying the same method to the space-time discretization of the compressible Navier-Stokes equations requires an additional stability constraint, the Von Neumann condition, which is more restrictive than the CFL condition in flow regions with small cell Reynolds numbers, i.e. boundary layers. Therefore, the Runge-Kutta method would no longer be a good smoother for the multigrid algorithm. Second, the multigrid algorithm itself should also be adapted since the equations are no longer hyperbolic but incompletely parabolic. In this paper, we will limit ourselves to finding an effective solver for viscous flows, to be combined with multigrid in our future work.

A possible solution to overcome the severe stability constraint in boundary layers, is the so-called implicit-explicit Runge-Kutta method, where the inviscid part is treated explicitly and the viscous part implicitly, such that only the CFL condition needs to be satisfied for stability. Contrary to Newton methods, this method does not require the Jacobian of the Euler flux but only of the viscous flux, the latter being readily available in the discretization [12]. However, in common with Newton methods, the implicit-explicit method does involve a global linear system. This rises the question whether the additional effort of solving the implicit system negates the relief of the stability constraint. To answer this question a priori is difficult as it highly depends on the case under consideration. Therefore, we will attempt to provide guidelines for aerodynamical applications based on representative numerical experiments.

Whether or not the implicit-explicit method significantly improves convergence in pseudo-time, it still involves a global sparse linear system and thus conflicts with the DG philosophy of locality. To preserve locality, we turn to the family of explicit Runge-Kutta methods derived by Kleb e.a. [13]. These schemes are specially designed for viscous flows and have stability domains which are much more stretched along the negative real axis than the RungeKutta schemes used for hyperbolic equations. Therefore, even though the Von Neumann condition still has to be satisfied for stability, it may no longer be the most restrictive. Since accuracy is not an issue in pseudo-time we can apply local pseudo-time stepping and combine the scheme developed for the Euler equations in [18], which is optimal in the inviscid regime, with the scheme presented in [13] for the viscous regime. By comparing the performance with the implicit-explicit scheme, we can see how effective this combination is in relieving the stability constraint for viscous flows.

The outline of this paper is the following. In Section 2, we briefly summarize the space-time discontinuous Galerkin discretization and give the weak formulation, the basis-functions and the resulting system of non-linear algebraic equations. The different pseudo-time stepping methods are described in 
Section 3 and their stability is analyzed using the scalar advection-diffusion equation as a model problem in Section 4. In Section 5, we compare the performance of both methods by computing steady and unsteady viscous flow around the NACA0012 airfoil and draw conclusions in Section 6.

\section{Summary of the space-time DG discretization}

This section summarizes the space-time discontinuous Galerkin discretization of the compressible Navier-Stokes equations as presented in [12], to which we refer for further details. The main idea is to consider the equations directly in the space-time domain using discontinuous basis-functions in space-time and introduce a numerical time-flux to ensure causality in time. The treatment of the viscous terms in $[3,4]$ was extended to the space-time context.

\subsection{Space-time formulation}

The space-time discontinuous Galerkin finite element method does not distinguish between space and time variables: the equations are considered in an open domain $\mathcal{E} \subset \mathbb{R}^{4}$, where a point with position $\bar{x}=\left(x_{1}, x_{2}, x_{3}\right)$ at time $t=x_{0}$ has Cartesian coordinates $\left(x_{0}, x_{1}, x_{2}, x_{3}\right)$. The flow domain $\Omega(t)$ at time $t$ is defined as $\Omega(t):=\left\{\bar{x} \in \mathbb{R}^{3}:(t, \bar{x}) \in \mathcal{E}\right\}$. Let $t_{0}$ and $T$ be the initial and final time of the evolution of the flow domain, then the space-time domain boundary $\partial \mathcal{E}$ consists of the hypersurfaces $\Omega\left(t_{0}\right):=\left\{x \in \mathcal{E}: x_{0}=t_{0}\right\}$, $\Omega(T):=\left\{x \in \mathcal{E}: x_{0}=T\right\}$, and $\mathcal{Q}:=\left\{x \in \partial \mathcal{E}: t_{0}<x_{0}<T\right\}$. Using this notation, the compressible Navier-Stokes equations can be written as:

$$
\begin{cases}U_{i, 0}+F_{i k}^{e}(U)_{, k}-\left(A_{i k r s}(U) U_{r, s}\right)_{, k}=0 & \text { on } \mathcal{E} \\ U=U_{0} & \text { on } \Omega\left(t_{0}\right), \\ U=\mathcal{B}\left(U, U^{b}\right) & \text { on } \mathcal{Q},\end{cases}
$$

with $U \in \mathbb{R}^{5}$ the vector of conservative variables, $F^{e} \in \mathbb{R}^{5 \times 3}$ the inviscid flux, $A \in \mathbb{R}^{5 \times 3 \times 5 \times 3}$ the homogeneity tensor, $U_{0} \in \mathbb{R}^{5}$ the initial flow field and $\mathcal{B} \in \mathbb{R}^{5}$ the boundary operator. The conservative variables, the inviscid flux and the viscous flux $F^{v} \in \mathbb{R}^{5 \times 3}$ are defined as:

$$
U=\left[\begin{array}{c}
\rho \\
\rho u_{j} \\
\rho E
\end{array}\right], \quad F_{k}^{e}=\left[\begin{array}{c}
\rho u_{k} \\
\rho u_{j} u_{k}+p \delta_{j k} \\
u_{k}(\rho E+p)
\end{array}\right], \quad F_{k}^{v}=\left[\begin{array}{c}
0 \\
\tau_{j k} \\
\tau_{k j} u_{j}-q_{k}
\end{array}\right],
$$


with $\rho$ the density, $\rho \vec{u}$ the momentum density vector, $\rho E$ the total energy density, $p$ the pressure, $\delta$ the Kronecker delta function, $\tau$ the shear stresses and $q$ the heat flux. The summation convention is used on repeated indices. The viscous flux $F^{v}$ is homogeneous with respect to the gradient of the conservative variables $\nabla U$. This defines the homogeneity tensor $A$ as:

$$
A_{i k r s}(U)=\frac{\partial F_{i k}^{v}(U, \nabla U)}{\partial\left(U_{r, s}\right)}
$$

This property is essential for the treatment of the viscous terms in the spacetime formulation of the compressible Navier-Stokes equations [12].

\subsection{Discretization}

The space-time discretization starts with the tessellation $\mathcal{T}_{h}^{n}=\{\mathcal{K}\}$ of the flow domain $\mathcal{E}$ in the time slab $\left(t_{n}, t_{n+1}\right)$. The associated functional spaces are defined as:

$$
\begin{gathered}
W_{h}:=\left\{W \in\left(L^{2}\left(\mathcal{E}_{h}\right)\right)^{5}:\left.W\right|_{\mathcal{K}} \circ G_{\mathcal{K}} \in\left(P^{k}(\hat{\mathcal{K}})\right)^{5}, \quad \forall \mathcal{K} \in \mathcal{T}_{h}\right\}, \\
V_{h}:=\left\{V \in\left(L^{2}\left(\mathcal{E}_{h}\right)\right)^{5 \times 3}:\left.V\right|_{\mathcal{K}} \circ G_{\mathcal{K}} \in\left(P^{k}(\hat{\mathcal{K}})\right)^{5 \times 3}, \quad \forall \mathcal{K} \in \mathcal{T}_{h}\right\},
\end{gathered}
$$

where $G_{\mathcal{K}}$ denotes the mapping of the master element $\hat{\mathcal{K}}=(-1,1)^{4}$ to element $\mathcal{K}$ and $P^{k}(\hat{\mathcal{K}})$ denotes the space of polynomials of degree at most $k$. Notice that $\nabla_{h} W_{h} \subset V_{h}$ where $\nabla_{h}$ is the broken gradient: $\left.\left(\nabla_{h} W_{h}\right)\right|_{\mathcal{K}}=\nabla\left(\left.W_{h}\right|_{\mathcal{K}}\right)$. The set of internal faces is denoted by $\mathcal{S}_{I}^{n}$ and the set of boundary faces by $\mathcal{S}_{B}^{n}$. The traces from the left and right are denoted by $(\cdot)^{L}$ and $(\cdot)^{R}$, respectively. The average operator is defined as $\left\{[\cdot\}=1 / 2\left((\cdot)^{L}+(\cdot)^{R}\right)\right.$ and the jump operator as $\llbracket \cdot \rrbracket_{k}=(\cdot)^{L} n_{k}^{L}+(\cdot)^{R} n_{k}^{R}$, with $n$ the outward normal vector of the element under consideration. Using this notation, the weak formulation of the compressible Navier-Stokes equations can be written as follows.

Find a $U \in W_{h}$, such that for all $W \in W_{h}$ :

$$
\begin{aligned}
& -\sum_{\mathcal{K} \in \mathcal{T}_{h}^{n}} \int_{\mathcal{K}}\left(W_{i, 0} U_{i}+W_{i, k}\left(F_{i k}^{e}-A_{i k r s} U_{r, s}+\mathcal{R}_{i k}\right)\right) d \mathcal{K} \\
& +\sum_{K \in \mathcal{T}_{h}^{n}}\left(\int_{K\left(t_{n+1}^{-}\right)} W_{i} U_{i}^{L} d K-\int_{K\left(t_{n}^{+}\right)} W_{i} U_{i}^{R} d K\right) \\
& +\sum_{\mathcal{S} \in \mathcal{S}_{I}^{n}} \int_{\mathcal{S}}\left(W_{i}^{L}-W_{i}^{R}\right) H_{i} d \mathcal{S}+\sum_{\mathcal{S} \in \mathcal{S}_{B}^{n}} \int_{\mathcal{S}} W_{i}^{L} H_{i}^{b} d \mathcal{S} \\
& -\sum_{\mathcal{S} \in \mathcal{S}_{I}^{n}} \int_{\mathcal{S}} \llbracket W_{i} \rrbracket_{k}\left\{\left\{A_{i k r s} U_{r, s}-\eta \mathcal{R}_{i k}^{\mathcal{S}}\right\} d \mathcal{S}\right. \\
& -\sum_{\mathcal{S} \in \mathcal{S}_{B}^{n}} \int_{\mathcal{S}} W_{i}^{L}\left(A_{i k r s}^{b} U_{r, s}^{b}-\eta \mathcal{R}_{i k}^{\mathcal{S}}\right) \bar{n}_{k}^{L} d \mathcal{S}=0 .
\end{aligned}
$$


Here, $H \in \mathbb{R}^{5}$ is the inviscid numerical flux from the HLLC approximate Riemann solver with the extension needed for moving meshes (cf. [18]) and $(\cdot)^{b}$ indicates dependence on the prescribed boundary data. The stability constant is $\eta>N_{f}$, with $N_{f}$ the number of faces per element. The local lifting operator is denoted by $\mathcal{R}^{\mathcal{S}} \in \mathbb{R}^{5 \times 3}$ and defined [12] as:

Find an $\mathcal{R}^{\mathcal{S}} \in V_{h}$, such that for all $V \in V_{h}$ :

$$
\sum_{\mathcal{K} \in \mathcal{T}_{h}^{n}} \int_{\mathcal{K}} V_{i k} \mathcal{R}_{i k}^{\mathcal{S}} d \mathcal{K}= \begin{cases}\int_{\mathcal{S}}\left\{V_{i k} A_{i k r s}\right\} \llbracket U_{r} \rrbracket_{s} d \mathcal{S} & \text { for } \mathcal{S} \in \mathcal{S}_{I}^{n}, \\ \int_{\mathcal{S}} V_{i k}^{L} A_{i k r s}^{L}\left(U_{r}^{L}-U_{r}^{b}\right) \bar{n}_{s} d \mathcal{S} & \text { for } \mathcal{S} \in \mathcal{S}_{B}^{n}\end{cases}
$$

The global lifting operator $\mathcal{R} \in \mathbb{R}^{5 \times 3}$ is obtained from the local lifting operator $\mathcal{R}^{\mathcal{S}}$ using the relation:

$$
\mathcal{R}=\sum_{\mathcal{S} \in \mathcal{S}_{I}^{n} \cup \mathcal{S}_{B}^{n}} \mathcal{R}^{\mathcal{S}}
$$

The upwind character of the numerical time-flux in the integrals over the time faces $K\left(t_{n}^{+}\right)$and $K\left(t_{n+1}^{-}\right)$ensures causality in time. The trial function $U$ and the test function $W$ in each element $\mathcal{K} \in \mathcal{T}_{h}^{n}$ are represented as polynomials:

$$
\left.U(t, \bar{x})\right|_{\mathcal{K}}=\hat{U}_{m} \psi_{m}(t, \bar{x}), \quad \text { and }\left.\quad W(t, \bar{x})\right|_{\mathcal{K}}=\hat{W}_{l} \psi_{l}(t, \bar{x}),
$$

with $(\hat{.})$ the expansion coefficients and $\psi$ the basis functions described in [12]. The system of algebraic equations for the expansion coefficients of $U$ is obtained by replacing $U$ and $W$ in the weak formulation with their polynomial expansions and using the fact that the test functions $W$ are arbitrary. For each physical time step the system can be written as:

$$
\mathcal{L}\left(\hat{U}^{n} ; \hat{U}^{n-1}\right)=0
$$

This paper focuses on solving system (1) using pseudo-time stepping methods. We add a pseudo-time derivative:

$$
\frac{\partial \hat{U}}{\partial \tau}=-\frac{1}{\Delta t} \mathcal{L}\left(\hat{U} ; \hat{U}^{n-1}\right)
$$

and iterate in pseudo-time $\tau$ to steady-state using Runge-Kutta methods. At steady-state we have $\hat{U}^{n}=\hat{U}$. In this paper we will investigate two different approaches:

(1) an implicit-explicit Runge-Kutta method, where the viscous terms are treated implicitly and the inviscid terms explicitly,

(2) a combination of two explicit Runge-Kutta schemes, one designed for inviscid flows and the other for viscous flows.

In our future work the most efficient of these methods will be used as a smoother in a full approximation multigrid scheme to enhance the overall efficiency of the method. 


\section{Pseudo-time stepping methods}

In this section, the different Runge-Kutta methods for the pseudo-time integration of system (2) are described.

First, we consider the explicit 5 stage Runge-Kutta method, which was successfully used to solve the system arising from the space-time discretization of the Euler equations in [18]. The method is derived from a second order 5 stage Runge-Kutta method using the correction proposed by Melson e.a. [15] to enhance the stability of the pseudo-time integration. For details of the derivation and the stability analysis for the Euler case we refer to [18]. This scheme is given by:

Algorithm 1 (EXI) Explicit Runge-Kutta method for inviscid flow with Melson correction.

(1) Initialize $\hat{V}^{0}=\hat{U}$.

(2) For all stages $s=1$ to 5 compute $\hat{V}^{s}$ as:

$$
\left(I+\alpha_{s} \lambda I\right) \hat{V}^{s}=\hat{V}^{0}+\alpha_{s} \lambda\left(\hat{V}^{s-1}-\mathcal{L}\left(\hat{V}^{s-1} ; \hat{U}^{n-1}\right)\right) .
$$

(3) Return $\hat{U}=\hat{V}^{5}$.

The Runge-Kutta coefficients at stage $s$ are denoted by $\alpha_{s}$ and defined as: $\alpha_{1}=0.0791451, \alpha_{2}=0.163551, \alpha_{3}=0.283663, \alpha_{4}=0.5$ and $\alpha_{5}=1.0$. The matrix $I$ represents the identity matrix. The coefficients were optimized to ensure rapid convergence to steady state. The factor $\lambda$ is the ratio between the pseudo-time step $\Delta \tau$ and the physical time step: $\lambda=\Delta \tau / \Delta t$. The Melson correction consists in treating $\hat{V}$ semi-implicitly, without this the scheme would become unstable for values of $\lambda$ around one.

Second, we consider the implicit-explicit version of the EXI method. The residual $\mathcal{L}$ defined in $(1)$ consist of two parts: $\mathcal{L}=\mathcal{L}^{e}+\mathcal{L}^{v}$, where $\mathcal{L}^{e}$ stems from the inviscid part of the compressible Navier-Stokes equations and $\mathcal{L}^{v}$ from the viscous part. The implicit-explicit method can be derived by introducing a Newton matrix $\mathcal{D}$, which approximates the Jacobian of the viscous part of the residual:

$$
\mathcal{D} \hat{V}^{s} \cong \mathcal{L}^{v}
$$

Here, the approximation consists of freezing the (non-linear) homogeneity tensor $A$ at the previous Runge-Kutta stage $s-1$. This approximation is relatively inexpensive compared with the Jacobian of the inviscid flux which would be required by a Newton solver, since $A$ is readily available in the discretization. The implicit-explicit Runge-Kutta method can thus be written as:

Algorithm 2 (IMEX) Implicit-explicit Runge-Kutta method. 
(1) Initialize $\hat{V}^{0}=\hat{U}$.

(2) For all stages $s=1$ to 5 compute $\hat{V}^{s}$ by solving:

$$
\left(I+\alpha_{s} \lambda(I+\mathcal{D})\right) \hat{V}^{s}=\hat{V}^{0}+\alpha_{s} \lambda\left((I+\mathcal{D}) \hat{V}^{s-1}-\mathcal{L}\left(\hat{V}^{s-1} ; \hat{U}^{n-1}\right)\right) .
$$

(3) Return $\hat{U}=\hat{V}^{5}$.

Note that the diffusive terms $\mathcal{L}^{v}$ in the residual $\mathcal{L}$ are not replaced by the approximation, both methods solve the same non-linear system $\mathcal{L}=0$. Clearly, the l.h.s. of the equation for $\hat{V}^{s}$ is no longer a diagonal matrix, but a global sparse block matrix, therefore $\hat{V}^{s}$ must be computed by solving the sparse linear system. We do so using the sparse iterative GMRES solver with Jacobi preconditioning, available in the PETSc package [17].

Finally, we consider one of the methods proposed by Kleb e.a. [13], which is an explicit 4 stage Runge-Kutta method, but with coefficients optimized for viscous flows:

Algorithm 3 (EXV) Explicit Runge-Kutta method for viscous flows.

(1) Initialize $\hat{V}^{0}=\hat{U}$.

(2) For all stages $s=1$ to 5 compute $\hat{V}^{s}$ as:

$$
\hat{V}^{s}=\hat{V}^{0}-\alpha_{s} \lambda \mathcal{L}\left(\hat{V}^{s-1} ; \hat{U}^{n-1}\right)
$$

(3) Return $\hat{U}=\hat{V}^{5}$.

For this method, the Runge-Kutta coefficients at stage $s$ are defined as: $\alpha_{1}=$ $0.0178571, \alpha_{2}=0.0568106, \alpha_{3}=0.174513$ and $\alpha_{4}=1$. A summary of the derivation of these values is given in Appendix A. With these coefficients, the stability domain of the Runge-Kutta method is very different from the one associated with the classic 4 stage Runge-Kutta method for inviscid flows. Notice that we do not apply the Melson correction to this scheme because we will not use it for values of $\lambda$ around one, for reasons which will become clear in the next section.

The EXI method is designed for inviscid flows, while the EXV method is designed for viscous flows. In aerodynamical applications, however, one encounters both flow regimes in the same simulation: the flow is inviscid in the far-field and viscous in boundary layers. Therefore, we will seek to combine both methods, based on their stability domains. The advantage of such a combination is that it remains local, contrary to the IMEX method which requires the solution of a global linear system. 


\section{Stability analysis}

The methods discussed in the previous section can all be applied to solve the system of non-linear equations (2) given by the space-time discretization of the compressible Navier-Stokes equations, provided a suitable pseudo-time stability constraint is satisfied. In this section, we derive these constraints.

\subsection{The model problem}

Rigorous stability analysis of numerical methods for the Navier-Stokes equations is extremely difficult and rarely attempted. Instead, in order to derive practical stability constraints, the method is required to be stable for the scalar advection-diffusion equation $[13,20]$ :

$$
u_{t}+a u_{x}=d u_{x x}, \quad t \in(0, T), \quad x \in \mathbb{R},
$$

with $a>0$ the advection constant and $d>0$ the diffusion constant. The domain is divided into uniform rectangular elements $\Delta t$ by $\Delta x$. The space-time discontinuous Galerkin method using the linear basis functions described in [12] gives the following discrete system for the vector of expansion coefficients $\hat{u}$ at time level $n$ :

$$
\mathcal{L}\left(\hat{u}^{n} ; \hat{u}^{n-1}\right)=0,
$$

with $\mathcal{L}=\mathcal{L}^{a}+\mathcal{L}^{d}$. The inviscid part $\mathcal{L}^{a}$ is defined as $\mathcal{L}^{a}\left(\hat{u}^{n} ; \hat{u}^{n-1}\right)=\mathcal{A} \hat{u}^{n}+$ $\mathcal{C} \hat{u}^{n-1}$ with $\mathcal{A}=\operatorname{blocktridiag}(A, B, 0)$ and $\mathcal{C}=\operatorname{blockdiag}(C)$. The matrices $A$, $B$ and $C$ depend on the Courant number:

$$
\sigma=\frac{a \Delta t}{\Delta x}
$$

and are given by:

$$
A=\left[\begin{array}{ccc}
-\sigma & -\sigma & \sigma \\
\sigma & \sigma & -\sigma \\
\sigma & \sigma & -\frac{4}{3} \sigma
\end{array}\right], \quad B=\left[\begin{array}{ccc}
1+\sigma & \sigma & -\sigma \\
-\sigma & \frac{1}{3}+\sigma & \sigma \\
-2-\sigma & -\sigma & 2+\frac{4}{3} \sigma
\end{array}\right]
$$

and

$$
C=\left[\begin{array}{ccc}
-1 & 0 & 0 \\
0 & -\frac{1}{3} & 0 \\
2 & 0 & 0
\end{array}\right]
$$


The viscous contribution $\mathcal{L}^{d}$ is defined as $\mathcal{L}^{d}\left(\hat{u}^{n}\right)=\mathcal{D} \hat{u}^{n}$ with $\mathcal{D}=\operatorname{blocktridiag}(D, E, F)$. The matrices $D, E$ and $F$ depend on the Von Neumann number:

$$
\delta=\frac{d \Delta t}{(\Delta x)^{2}}
$$

as well as on the stabilization constant $\eta$ :

$$
D=\delta\left[\begin{array}{ccc}
-2 \eta & 1-2 \eta & 2 \eta \\
-1+2 \eta & -2+2 \eta & 1-2 \eta \\
2 \eta & -1+2 \eta & -\frac{13}{6} \eta
\end{array}\right], \quad E=\delta\left[\begin{array}{ccc}
4 \eta & 0 & -4 \eta \\
0 & 4 \eta & 0 \\
-4 \eta & 0 & \frac{13}{3} \eta
\end{array}\right]
$$

and

$$
F=\delta\left[\begin{array}{ccc}
-2 \eta & -1+2 \eta & 2 \eta \\
1-2 \eta & -2+2 \eta & -1+2 \eta \\
2 \eta & 1-2 \eta & -\frac{13}{6} \eta
\end{array}\right]
$$

The system of algebraic equations (3) resulting from the space-time discontinuous Galerkin discretization of the model problem is solved using the pseudotime stepping methods described in the previous section. Since the stability in pseudo-time of the Runge-Kutta methods is only affected by the transients, we only consider the homogeneous part $(\mathcal{C}=0)$ of the linear system $(3)$. Thus, the pseudo-time equation for the model problem becomes:

$$
\frac{\partial \hat{u}^{n}}{\partial \tau}=-\frac{1}{\Delta t}(\mathcal{A}+\mathcal{D}) \hat{u}^{n} .
$$

\subsection{Stability of the EXI and EXV method}

The stability analysis of the EXI and EXV method is similar and therefore treated simultaneously in this section. We begin by noticing that the matrix $\mathcal{A}+\mathcal{D}$ can be diagonalized as $Q M Q^{-1}$, with $Q$ the matrix of right eigenvectors of $\mathcal{A}+\mathcal{D}$ and $M$ the diagonal matrix with the (complex) eigenvalues $\mu$. Using this property and introducing the new vector $w=Q^{-1} \hat{u}^{n}$, reduces equation (6) to the simple scalar test model:

$$
\frac{\partial w}{\partial \tau}=-\frac{\mu}{\Delta t} w
$$

for all eigenvalues $\mu$ of $\mathcal{A}+\mathcal{D}$. When applying the EXI method to this model equation, the Runge-Kutta stages $w^{s}$ are computed as:

$$
\left(1+\alpha_{s} \lambda\right) w^{s}=w^{0}+\alpha_{s} \lambda(1-\mu) w^{s-1}
$$


with $\lambda=\Delta \tau / \Delta t$ and for the EXV as:

$$
w^{s}=w^{0}-\alpha_{s} \lambda \mu w^{s-1} .
$$

Using these equations the relation between two consecutive pseudo-time steps can easily be derived and is written in generic form as:

$$
w^{n}=G(-\lambda \mu) w^{n-1}
$$

with $G$ the algorithm dependent amplification factor. In stability analysis, we are interested in the behavior of a perturbation of the initial condition (see for example [20]). Due to linearity, the equation for the perturbation is the same as the equation for $w$ and after $n$ steps we obtain:

$$
w^{n}=G(-\lambda \mu)^{n} w^{i},
$$

with $w^{i}$ the initial solution. Clearly, the perturbation $w$ is bounded if $\left\|G^{n}\right\|$ is bounded, where $\|\cdot\|$ denotes the Euclidian (or discrete $l_{2}$ ) norm $[11,20]$. Therefore, a sufficient condition for stability is that all values $-\lambda \mu$ must lie inside the stability domain $S$ given by:

$$
S=\{z \in \mathbb{C}:|G(z)| \leq 1\}
$$

Remember that the discretization of the advection-diffusion equation only depends on the Courant number (4), the Von Neumann number (5) and the constant $\eta$. For given values of these numbers, the factor $\lambda$ of the Runge-Kutta algorithm should be chosen such that $-\lambda \mu$ lies inside the stability domain $S$ for all $\mu$. Once a suitable $\lambda$ is found, it is convenient to express the stability in terms of the pseudo-time Courant and Von Neumann numbers: $\sigma_{\Delta \tau}=\lambda \sigma$ and $\delta_{\Delta \tau}=\lambda \delta$. For stability, the pseudo-time step $\Delta \tau$ must satisfy the CourantFriedrichs-Levy (CFL) condition and the Von Neumann condition:

$$
\Delta \tau \leq \frac{\sigma_{\Delta \tau} \Delta x}{a} \text { and } \quad \Delta \tau \leq \frac{\delta_{\Delta \tau}(\Delta x)^{2}}{d}
$$

We distinguish between flow regimes by introducing the cell Reynolds number, defined as:

$$
\operatorname{Re}_{\Delta x}=\frac{a \Delta x}{d} .
$$

In aerodynamical computations, the flow is inviscid in most of the domain, yet significant viscous effects occur in the boundary layer near the airfoil. Therefore we will consider the following regimes:

(1) Steady-state, inviscid: $\sigma=100$ and $\operatorname{Re}_{\Delta x}=100$,

(2) Steady-state, viscous: $\sigma=100$ and $\operatorname{Re}_{\Delta x}=0.01$,

(3) Time-dependent, inviscid: $\sigma=1$ and $\operatorname{Re}_{\Delta x}=100$,

(4) Time-dependent, viscous: $\sigma=1$ and $\operatorname{Re}_{\Delta x}=0.01$. 
The Von Neumann condition can be expressed in terms of the cell Reynolds number as:

$$
\Delta \tau \leq \frac{\delta_{\Delta \tau} \operatorname{Re}_{\Delta x} \Delta x}{a} .
$$

Thus, for the inviscid flow regime the CFL condition is the most restrictive, for the viscous flow regime the Von Neumann condition and the threshold between both is given by $\delta_{\Delta \tau} \operatorname{Re}_{\Delta x}=\sigma_{\Delta \tau}$.

The stability domains of the EXI and EXV method and the values $-\lambda \mu$ are plotted in Figures 1, 2, 3 and 4. For inviscid flow regimes with pseudo-time Courant number around $\alpha_{\Delta \tau}=1.7$, the EXI method is stable and the EXV is unstable, but for viscous flow regimes with pseudo-time Von Neumann number $\delta_{\Delta \tau}=0.8$, the converse holds. Stability constraints for which both methods are stable are given in Table 1, confirming that the EXI method is preferable in the inviscid regime and the EXV in the viscous regime. Therefore, we combine the EXI and EXV by looking at the cell Reynolds number, and, for that particular cell, deploy whichever scheme has the mildest stability restriction. We can apply this type of local pseudo-time stepping because accuracy is not an issue in pseudo-time.

The Melson correction is applied to the EXI scheme to ensure stability for for values of $\lambda$ around one, which is the case for the time-dependent inviscid flow regime (Figure 3). For all other flow regimes, $\lambda$ is small and the Melson correction vanishes. Since we only apply the EXV scheme in the viscous flow regime, the Melson correction is unnecesary for this scheme.

\subsection{Stability of the IMEX method}

The IMEX method solves the inviscid part of the equations with the EXI method and treats the viscous part implicitly. The main idea is that the stability should now only depend on the inviscid part, so only the CFL condition needs to be satisfied, thereby allowing the EXI method to be deployed for both the inviscid and viscous flow regimes. Unfortunately, the matrices $\mathcal{A}$ and $\mathcal{D}$ in (6) do not commute, making it impossible to obtain a scalar model problem through diagonalization, as was done for the explicit method. Stability analysis of IMEX methods for general non-commuting matrices is still largely an open problem, although recently, for the related W-methods, results have been presented by Ostermann [16]. In this section, we will proof stability of the IMEX method by directly estimating the norm of the amplification factor G.

For the IMEX method the Runge-Kutta stages $\hat{v}^{s}$ are computed by solving 
the sparse linear system:

$$
\left(I+\alpha_{s} \lambda(I+\mathcal{D})\right) \hat{v}^{s}=\hat{v}^{0}+\alpha_{s} \lambda(I-\mathcal{A}) \hat{v}^{s-1} .
$$

The starting point of our analysis is the fact that $\mathcal{D}$ is a Hermitian matrix: $\mathcal{D}=Q M Q^{T}$ where $Q$ is a unitary matrix and $M$ the diagonal matrix with the (real and positive) eigenvalues $\mu$ of $\mathcal{D}$. For a unitary matrix $Q^{-1}=Q^{T}$ and the l.h.s. of (9) can be written as:

$$
\begin{aligned}
I+\alpha_{s} \lambda(I+\mathcal{D}) & =Q\left(I+\alpha_{s} \lambda(I+M)\right) Q^{T} \\
& =Q M_{s} Q^{T},
\end{aligned}
$$

with $M_{s}$ the diagonal matrix with values $1+\alpha_{s} \lambda(1+\mu)$. Introducing the decomposition (10) into (9) gives:

$$
\begin{aligned}
M_{s} w^{s} & =w^{0}+\alpha_{s} \lambda Q^{T}(I-\mathcal{A}) Q w^{s-1}, \\
& =w^{0}+\alpha_{s} \lambda P_{\mathcal{A}} w^{s-1}
\end{aligned}
$$

with $w^{s}=Q^{T} \hat{v}^{s}$ and $P_{\mathcal{A}}=Q^{T}(I-\mathcal{A}) Q$. Therefore, the relation between two consecutive pseudo-time steps is: $w^{n}=G w^{n-1}$ with the amplification matrix $G$ defined as:

$$
\begin{gathered}
G=M_{5}^{-1}\left(I+\alpha_{5} \lambda P_{\mathcal{A}}\right. \\
M_{4}^{-1}\left(I+\alpha_{4} \lambda P_{\mathcal{A}}\right. \\
\quad \cdots \\
\left.\left.\quad M_{1}^{-1}\left(I+\alpha_{1} \lambda P_{\mathcal{A}}\right)\right)\right) .
\end{gathered}
$$

If $\|G\| \leq 1$, then $\left\|G^{n}\right\| \leq 1$ and the method is stable. Our stability analysis aims at a direct estimation of this norm, therefore we consider the following upper bound:

$$
\begin{aligned}
& \|G\| \leq\left\|M_{5}^{-1}\right\|\left(1+\alpha_{5} \lambda\left\|P_{\mathcal{A}}\right\|\right. \\
& \left\|M_{4}^{-1}\right\|\left(1+\alpha_{4} \lambda\left\|P_{\mathcal{A}}\right\|\right. \\
& \quad \cdots \\
& \left.\left.\quad\left\|M_{1}^{-1}\right\|\left(1+\alpha_{1} \lambda\left\|P_{\mathcal{A}}\right\|\right)\right)\right) .
\end{aligned}
$$

The matrices $M_{s}^{-1}$ are equal to:

$$
M_{s}^{-1}=\operatorname{diag}\left(\frac{1}{1+\alpha_{s} \lambda\left(1+\mu_{1}\right)}, \cdots, \frac{1}{1+\alpha_{s} \lambda\left(1+\mu_{n}\right)}\right),
$$

with $\mu$ the eigenvalues of $\mathcal{D}$. The Euclidian norm of $M_{s}^{-1}$ can be estimated as:

$$
\left\|M_{s}^{-1}\right\|=\max _{i \in\{1, \cdots, n\}} \frac{1}{1+\alpha_{s} \lambda\left(1+\mu_{i}\right)}<\frac{1}{1+\alpha_{s} \lambda},
$$


since $\mu, \alpha_{s}, \lambda>0$. Using this estimation, the upper bound for the Euclidian norm of $G$ is then provided by the following estimate:

$$
\begin{aligned}
\|G\| \leq \frac{1}{1+\alpha_{5} \lambda}\left(1+\alpha_{5} \lambda\left\|P_{\mathcal{A}}\right\|\right. \\
\frac{1}{1+\alpha_{4} \lambda}\left(1+\alpha_{4} \lambda\left\|P_{\mathcal{A}}\right\|\right. \\
\cdots \\
\left.\left.\frac{1}{1+\alpha_{5} \lambda}\left(1+\alpha_{1} \lambda\left\|P_{\mathcal{A}}\right\|\right)\right)\right) .
\end{aligned}
$$

The r.h.s. of this equation is called the stability function, denoted by $f\left(\lambda,\left\|P_{\mathcal{A}}\right\|\right)$ and plotted for $\left\|P_{\mathcal{A}}\right\|=1$ in Figure 5. If $\left\|P_{\mathcal{A}}\right\|<1$ we find ourselves below the curve in Figure 5, therefore: $\left\|P_{\mathcal{A}}\right\| \leq 1 \Rightarrow f\left(\lambda,\left\|P_{\mathcal{A}}\right\|\right) \leq 1 \Rightarrow\|G\| \leq 1$ meaning $\left\|P_{\mathcal{A}}\right\| \leq 1$ is a sufficient condition for stability of the implicit-explicit method. Since the matrix $P_{\mathcal{A}}$ is defined as $P_{\mathcal{A}}=Q^{T}(I-\mathcal{A}) Q$, with $Q$ a unitary matrix (hence $\|Q\|=1$ ), this implies that the stability of the IMEX method is only determined by the following condition:

$$
\|I-\mathcal{A}\| \leq 1
$$

Since $\mathcal{A}$ only depends on the Courant number (4), this condition implies that the IMEX method is stable independently of the Von Neumann number, and only the CFL condition has to be satisfied.

The fact that the IMEX method does not need the Von Neumann condition for stability is a considerable advantage over fully explicit methods. But does this advantage outweight the additional effort of solving the implicit linear system? The answer to this question highly depends on the case under consideration. Therefore, we will adress it in the following section through numerical experiments.

\section{Results}

In this section, two benchmark problems are considered. We present the results obtained with the space-time discontinuous Galerkin method combined with either the explicit or implicit-explicit pseudo-time stepping methods.

To test the performance of the pseudo-time stepping methods, we have chosen the cases A1 and A7 described in [7] for the viscous flow past a NACA0012 airfoil. The Prandtl number is fixed at $\mathrm{Pr}=0.72$ for both cases while the far-field Mach and Reynolds numbers and the angle of attack are given by:

A1. $\mathrm{M}_{\infty}=0.8, \operatorname{Re}_{\infty}=73$ and $\alpha=12^{\circ}$ : steady-state viscous flow. 
A7. $\mathrm{M}_{\infty}=0.85, \mathrm{Re}_{\infty}=10^{4}$ and $\alpha=0^{\circ}$ : time-dependent viscous flow with a shock and vortex shedding.

The A1 case has become a standard benchmark for discontinuous Galerkin methods for the compressible Navier-Stokes equations as it was treated in the seminal paper by Bassi and Rebay [3].

For laminar viscous flow, the boundary layer at the nose of the airfoil is usually estimated as:

$$
b \approx 5 / \sqrt{\operatorname{Re}_{\infty}}
$$

which means that $b \approx 0.6$ in the A1 case and $b \approx 0.05$ in the A7 case. To compute the boundary layer in the A7 case with reasonable accuracy, we have chosen a C-type grid for viscous flows with $224 \times 76$ elements which offers more than 30 elements in the $b \approx 0.05$ boundary layer, see Figure 6 . For the A1 case, the boundary layer is much thicker and we can use a coarsened grid with $112 \times 38$ elements. In both cases, we use linear basis functions.

The space-time method is unconditionally stable in physical time, which allows us to take any physical time step $\Delta t$ and solve the non-linear system using the pseudo-time stepping methods. For the steady-state case, we take one huge time step $\Delta t=10^{10}$ and for the unsteady case we take time steps $\Delta t=0.05$ which follows from the physical CFL constraint needed to capture the vortex shedding. Since accuracy is not an issue in pseudo-time we can use local steps $(\Delta \tau)_{K}$, which are determined for each element $K$ as:

$$
(\Delta \tau)_{K}= \begin{cases}\min \left\{(\Delta \tau)_{K}^{e},(\Delta \tau)_{K}^{v}\right\} & \text { for EXI and EXV } \\ (\Delta \tau)_{K}^{e} & \text { for IMEX }\end{cases}
$$

The local inviscid and viscous pseudo-time steps are computed as:

$$
\begin{array}{ll}
(\Delta \tau)_{K}^{e}=\frac{\sigma_{\Delta \tau} d_{K}}{\lambda_{K}^{e}} \quad \text { with } \quad \lambda_{K}^{e}=\max \left\{\left|u_{K}\right|-a_{K},\left|u_{K}\right|+a_{K}\right\}, \\
(\Delta \tau)_{K}^{v}=\frac{\delta_{\Delta \tau}\left(d_{K}\right)^{2}}{\lambda_{K}^{v}} \quad \text { with } \quad \lambda_{K}^{v}=\max \left\{\frac{1}{c_{v}} \frac{\kappa_{K}}{\rho_{K}}, \frac{4}{3} \frac{\mu_{K}}{\rho_{K}}\right\},
\end{array}
$$

where $\sigma_{\Delta \tau}$ is the pseudo-time Courant number, $\delta_{\Delta \tau}$ the pseudo-time Von Neumann number (both from Table 1) and $d_{K}$ the diameter of the circle inscribed in element $K$. The cell Reynolds number $\operatorname{Re}_{K}$ is defined as:

$$
\operatorname{Re}_{K}=\frac{\lambda_{K}^{e} d_{K}}{\lambda_{K}^{v}}
$$

and $\lambda^{e}$ represents the maximum of the absolute value of the eigenvalues of the inviscid Jacobian and $\lambda^{v}$ of the viscous Jacobian. Furthermore, $u_{K}$ is the flow speed, $a_{K}$ the speed of sound and $\rho_{K}$ the density in element $K$. The specific heat at constant volume $c_{v}$ is constant throughout the domain but the 
dynamic viscosity $\mu_{K}$ and the thermal conductivity coefficient $\kappa_{K}$ depend on the temperature in element $K$, see $[12,14]$. Even though the stability analysis was only done for the advection-diffusion equation on a periodic domain, the resulting stability constraints proved also adequate for the A1 and A7 case. The local Mach number isolines for both cases are presented in Figure 7, the convergence results for the A1 case in Figure 8 and for the A7 case in Figure 9 .

In the A1 case, the cell Reynolds number varies between 0.09 and 88 which explains why the convergence of the EXI method is very slow: one order of convergence in 80000 pseudo-time iterations. If the EXV method is applied for elements with $\operatorname{Re}_{K}<1$, one order of convergence requires ten times less iterations and seven orders of convergence are reached within 50000 steps. In terms of iterations, the IMEX method performs much better: in this case six orders of convergence in 3000 pseudo-time steps. However, due to the construction and solution of the implicit system the work load per pseudotime step is much higher. In an effort to quantify this additional cost, we define the basic work unit as the work needed to perform one explicit Runge-Kutta step. For each Runge-Kutta stage, the implicit linear system must be solved which is difficult because the matrix is neither symmetric nor positive definite. Using the sparse GMRES solver with Jacobi preconditioner available in the PETSc package [17], we found that the system is typically solved in about 80 iterations. This translates to about 25 work units for an implicit-explicit Runge-Kutta step, based on CPU-time measurements. The IMEX method is still significantly faster than the EXI but the combination between EXI and EXV is clearly the fastest.

In the A7 case, for each physical-time step, the EXI method achieves three orders of convergence in 1000 pseudo-time steps, see Figure 9. The physical time-step is already fairly small in order to capture the vortex shedding, which explains the relatively small number of pseudo-time steps needed to solve the system. In this case, the cell Reynolds number varies between 2.5 and 14000 and if the EXV method is applied for elements with $\mathrm{Re}_{K}<10$ the convergence is twice as fast. The IMEX method requires 200 iterations, which is two and half times faster than the combined EXI and EXV method. However, at a twenty-five times higher cost per iteration, the IMEX method is the slowest when expressed in work units.

\section{Discussion and conclusions}

When applying the space-time discontinuous Galerkin method to the compressible Navier-Stokes equations one obtains a system of non-linear algebraic equations. To solve this system we presented two pseudo-time stepping meth- 
ods: the combined EXI and EXV method and the IMEX method.

The EXI and EXV method both treat the inviscid and viscous terms explicitly. We showed that these algorithms are stable if either the pseudo-time CFL or the Von Neumann condition is satisfied, depending on the cell Reynolds number. In the inviscid flow regime, the cell Reynolds numbers are high and the CFL condition is the most restrictive, while in the viscous flow regime low cell Reynolds numbers occur so that the Von Neumann condition is most restrictive. We also considered the implicit-explicit version of the EXI method where the viscous terms are treated implicitly and the inviscid terms explicitly. We showed that the stability of the IMEX method only depends on the inviscid part, thereby effectively relieving us of the Von Neumann condition. The price to pay for this advantage is the construction and solution of a global sparse linear system. The question whether the advantage of not having to satisfy the Von Neumann condition outweighs this disadvantage was answered for two numerical experiments where both the viscous and inviscid flow regime occur in the same simulation, albeit in different proportions. We compared the performance of the different Runge-Kutta methods and arrive at the following guidelines for aerodynamical simulations:

(1) The best option is to use the EXI in the inviscid part of the flow domain together with the EXV in the viscous part. With local pseudo-time stepping, the cell Reynolds number determines which scheme to use in which cell, based on the given stability constraints.

(2) The IMEX method is very well suited for both the inviscid part and the viscous part when expressed in terms of iterations. However, the work load per iteration is such that this method only gives significant gain in terms of work load for fairly viscous flows, this gain still being less than the one obtained with the combined explicit methods.

We showed that pseudo-time stepping with local explicit methods efficiently solves the system of non-linear algebraic equations. It is not necessary to give up locality for improved stability as was done with the implicit-explicit method. In our future work, we will focus on further reducing the computational effort by applying the combined explicit methods as a smoother in a multigrid algorithm.

\section{Acknowledgements}

The advice and support of Dr. A. Bell concerning the PETSc package and its sparse linear solvers is greatly appreciated. This research has been conducted in the STW project TWI.5541, entitled Advanced simulation techniques for vortex dominated flows in aerodynamics. The financial support from STW 
and the National Aerospace Laboratory NLR is gratefully acknowledged.

\section{A Details of EXV method}

In [13] a family of Runge-Kutta schemes for efficient time-marching of viscous flow problems is presented. We used a member of this family, the EXV method, for local pseudo-time stepping in flow regions with low cell Reynolds numbers. In this appendix, we summarize the derivation of the entire family.

Consider the following $N$ stage Runge-Kutta scheme:

(1) Initialize $\hat{v}^{0}=\hat{u}$.

(2) For all stages $s=1$ to $N$ compute $\hat{v}^{s}$ as:

$$
\hat{v}^{s}=\hat{v}^{0}-\alpha_{s} \lambda \mathcal{L}\left(\hat{v}^{s-1} ; \hat{u}^{n-1}\right) .
$$

(3) Return $\hat{u}=\hat{v}^{N}$.

When applied to the simple model problem:

$$
\frac{\partial u}{\partial \tau}=-\frac{\mu}{\Delta t} u
$$

the stages $s$ are updated according to: $v^{s}=v^{0}-\alpha_{s} \lambda \mu v^{s-1}$ and therefore the amplification factor $G$ is of the form:

$$
G_{N}(z)=1+\alpha_{N} z+\alpha_{N} \alpha_{N-1} z^{2}+\cdots+\alpha_{N} \cdots \alpha_{1} z^{N}
$$

with $z=-\lambda \mu \in \mathbb{C}$. The family of Runge-Kutta schemes proposed in [13] can be derived by chosing the coefficients $\alpha_{s}$ in such a way that the amplification factor equals Manteuffel's transformation of Tchebyshev polynomials:

$$
G_{N}(z)=\frac{T_{N}((d-z) / \epsilon)}{T_{N}(d / \epsilon)},
$$

where $T_{N}$ denotes the $N$-th Tchebyshev polynomial defined recursively as:

$$
T_{n+1}(z)=2 z T_{n}(z)-T_{n-1}, \quad n \in \mathbb{N},
$$

with $T_{0}(z)=1$ and $T_{1}(z)=z$. Here, the parameter $d$ defines the family of $N$ stage Runge-Kutta schemes and the parameter $\epsilon$ is chosen such that:

$$
G_{N}(0)=1 \quad \text { and }\left.\quad \frac{d G_{N}}{d z}\right|_{z=0}=1
$$


which ensures that the stability region touches the imaginary axis and is symmetric w.r.t. the real axis. The parameter $d$ controls the scaling of the stability region.

The family member used in this paper is the 4-stage Runge-Kutta scheme with $d=-14$. For this scheme, we use the fourth order Tchebyshev polynomial: $T_{4}(z)=8 z^{4}-8 z^{2}+1$ and obtain the following stability region:

$$
G_{4}(z)=1+\frac{16 \epsilon^{2} d-32 d^{3}}{D} z+\frac{48 d^{2}-8 \epsilon^{2}}{D} z^{2}-\frac{32 d}{D} z^{3}+\frac{8}{D} z^{4}
$$

with $D=8 d^{4}-8 d^{2} \epsilon^{2}+\epsilon^{4}$. The coefficients $\alpha_{s}$ can now be computed by equating (A.2) with (A.1) which gives:

$$
\alpha_{1}=-\frac{1}{4 d}, \quad \alpha_{2}=\frac{4 d}{\epsilon^{2}-6 d^{2}}, \quad \alpha_{3}=\frac{6 d^{2}-\epsilon^{2}}{2 d\left(\epsilon^{2}-2 d^{2}\right)}, \alpha_{4}=\frac{16 d\left(\epsilon^{2}-2 d^{2}\right)}{D} .
$$

The condition $G_{4}(0)=1$ is already satisfied and the condition on the derivative of $G$ becomes:

$$
\frac{16 \epsilon^{2} d-32 d^{3}}{D}=1
$$

which has four solutions for $\epsilon$ from which we choose the following:

$$
\epsilon=\sqrt{4 d(d+2)-2 \sqrt{16 d^{2}+8 d^{3}+2 d^{4}}} .
$$

In the same way, we can derive the other members of the family. Note, however, that only even $N$ produces consistent schemes.

\section{References}

[1] D. Arnold, F. Brezzi, B. Cockburn, and D. Marini. Unified analysis of discontinuous Galerkin methods for elliptic problems. SIAM J. Numer. Anal., 39:1749-1779, 2002.

[2] F. Bassi, A. Crivellini, S. Rebay, and M. Savini. Discontinuous Galerkin solution of the Reynolds-averaged Navier-Stokes and $k-\omega$ turbulence model equations. Computers \& Fluids, 34(4-5):507-540, 2004.

[3] F. Bassi and S. Rebay. A high-order accurate discontinuous finite element method for the numerical solution of the compressible Navier-Stokes equations. J. Comput. Phys., 131:267-279, 1997.

[4] F. Bassi and S. Rebay. Numerical evaluation of two discontinuous Galerkin methods for the compressible Navier-Stokes equations. Int. J. Num. Meth. Fluids, 40:197-207, 2002. 
[5] C.E. Baumann and J.T. Oden. A discontinuous $h p$ finite element method for the Euler and Navier-Stokes equations. Tenth International Conference on Finite Elements in Fluids (Tucson, AZ, 1998). Internat. J. Numer. Methods Fluids, 31(1):79-95, 1999.

[6] F. Brezzi, G. Manzini, D. Marini, P. Pietra, and A. Russo. Discontinuous Galerkin approximations for elliptic problems. Numer. Meth. Part. Diff. Eq., 16(4):365-378, 2000.

[7] M.O. Bristeau, R. Glowinski, J.Periaux, and H. Viviand (Eds.). Numerical simulation of compressible Navier-Stokes flows: A GAMM workshop, 4-6 December 1985, Nice, France. Braunschweig, Vieweg, 1987.

[8] B. Cockburn and C.-W. Shu. The local discontinuous Galerkin method for timedependent method for convection-diffusion systems. SIAM J. Numer. Anal., 35:2240-2463, 1998.

[9] B. Cockburn and C.-W. Shu. Runge-Kutta discontinuous Galerkin methods for convection-dominated problems. J. Sci. Comput., 16(3):173-261, 2001.

[10] V. Dolejší. On the discontinuous Galerkin method for the numerical solution of the Navier-Stokes equations. Int. J. Numer. Meth. Fluids, 45:1083-1106, 2004.

[11] E. Hairer and G. Wanner. Solving ordinary differential equations II. Stiff and differential-algebraic problems, Second revised edition. Springer Verlag, Berlin, 2002.

[12] C.M. Klaij, J.J.W. van der Vegt, and H. van der Ven. A space-time discontinuous Galerkin discretization for the compressible Navier-Stokes equations. submitted to J. Comput. Phys., see also Technical Memorandum 1774 (http://www.math.utwente.nl/publications), 2005.

[13] W.L. Kleb, W.A. Wood, and B. van Leer. Efficient Multi-Stage Time Marching for Viscous Flows via Local Preconditioning. AIAA J., 99-3267:181-194, 1999.

[14] J. Kok. An Industrially Applicable Solver for Compressible, Turbulent Flows. PhD thesis, Delft University of Technology, Delft, The Netherlands, 1998.

[15] N.D. Melson, M.D. Sanetrik, and H.L. Atkins. Time-accurate Navier-Stokes calculations with multigrid acceleration. In Proc. 6th Copper Mountain Confer. on Multigrid Methods, 1993.

[16] A. Ostermann. Stability of W-methods with applications to operator splitting and to geometric theory. Appl. Num. Math., 42:353-366, 2002.

[17] PETSc package. http://www-unix.mcs.anl.gov/petsc/petsc-2.

[18] J.J.W. van der Vegt and H. van der Ven. Space-time discontinuous Galerkin finite element method with dynamic grid motion for inviscid compressible flows. I. General formulation. J. Comput. Phys, 182:546-585, 2002. 
[19] H. van der Ven and J.J.W. van der Vegt. Space-time discontinuous Galerkin finite element method with dynamic grid motion for inviscid compressible flows. II. Efficient flux quadrature. Comput. Meth. Appl. Mech. Engrg., 191:47474780, 2002.

[20] Pieter Wesseling. Principles of Computational Fluid Dynamics. Springer, 2000. 

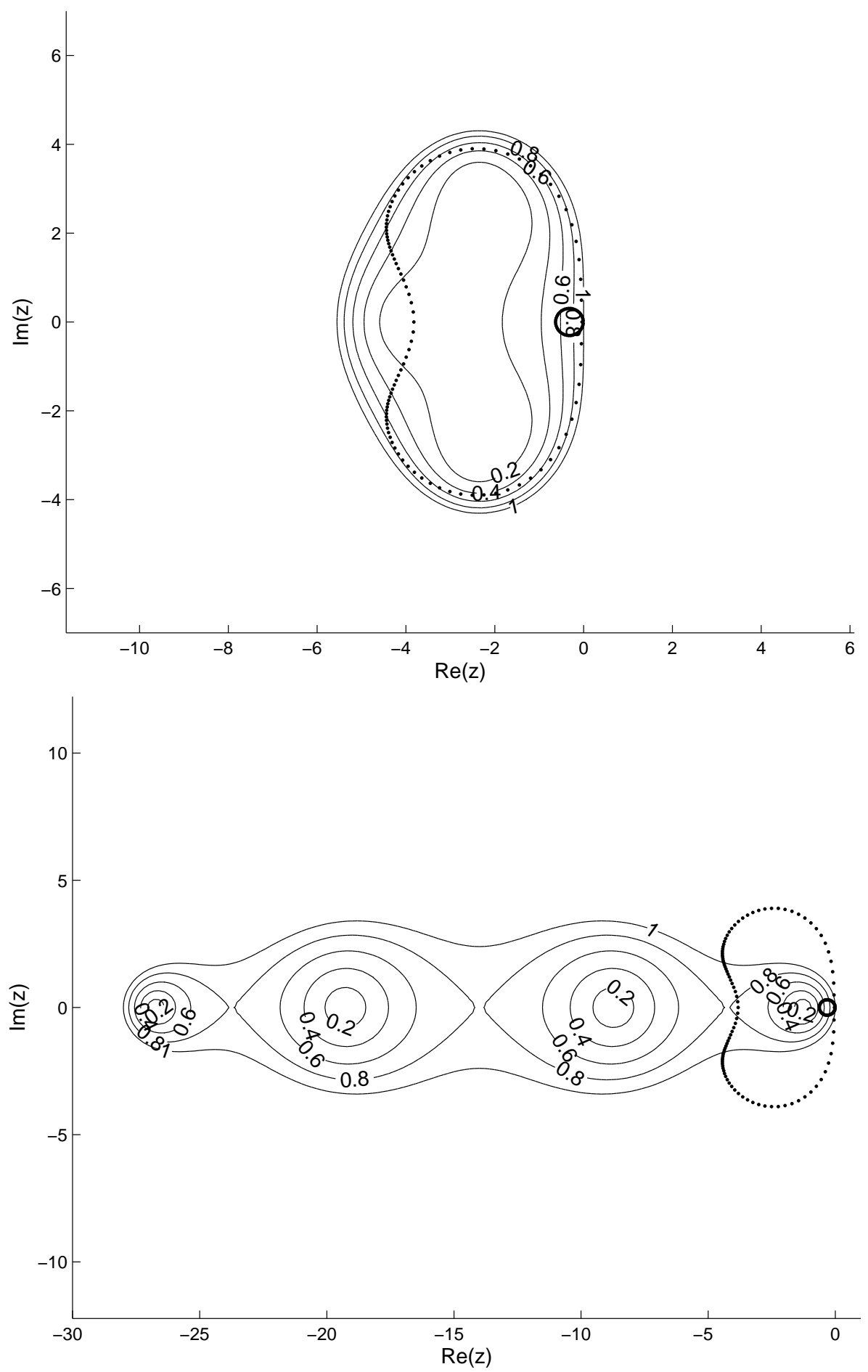

Fig. 1. The stability domain $S$ and values $-\lambda \mu$ (dots) for the EXI method (top) and EXV method (bottom) in the steady-state inviscid flow regime with $\lambda=1.8 \cdot 10^{-2}$. The pseudo-time CFL number is 1.8 and for this constraint only the EXI method is stable. 

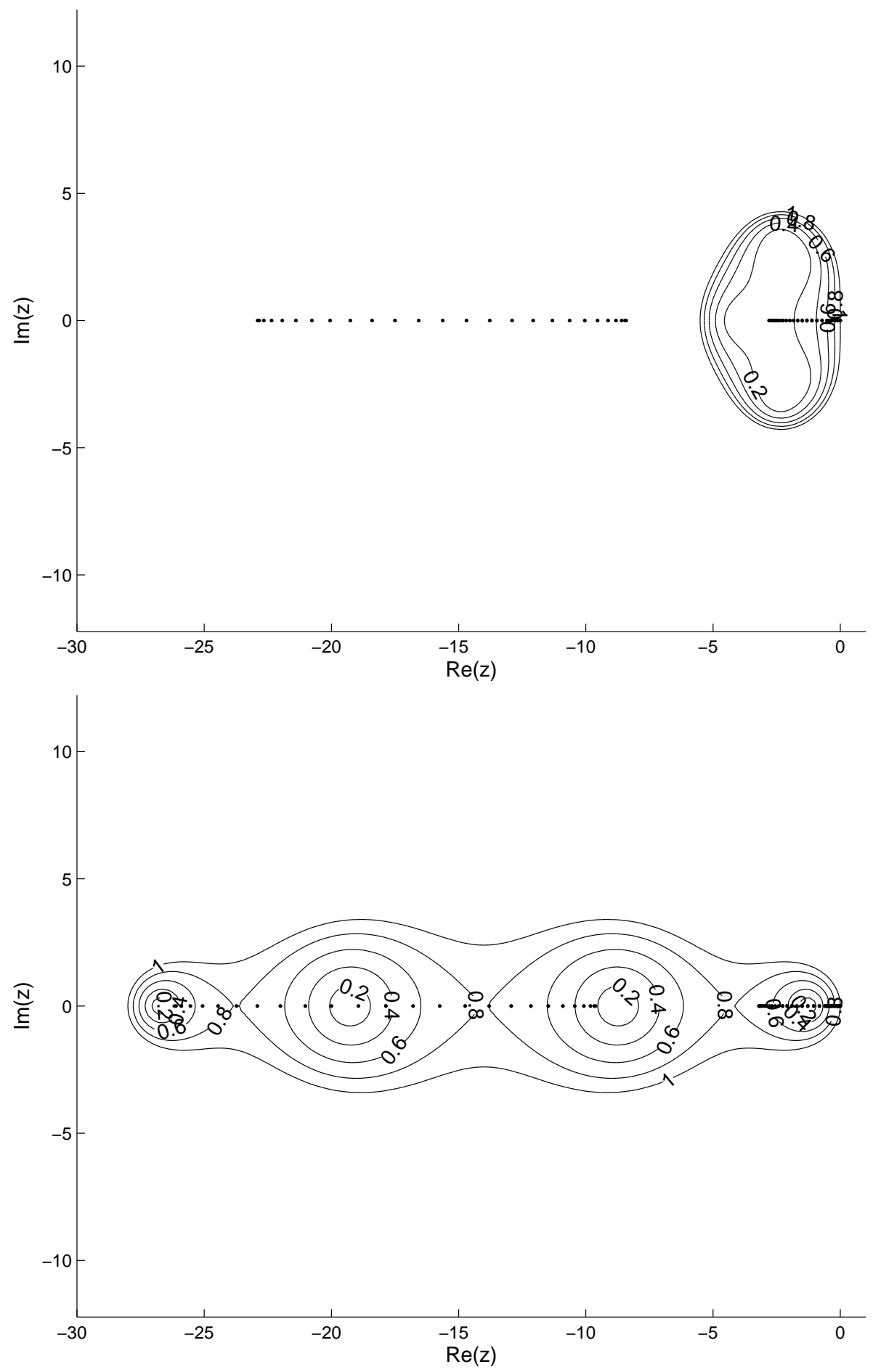

Fig. 2. The stability domain $S$ and values $-\lambda \mu$ (dots) for the EXI method (top) and EXV method (bottom) in the steady-state viscous flow regime with $\lambda=8 \cdot 10^{-5}$. The pseudo-time Von Neumann number is 0.8 and for this constraint only the EXV method is stable. 

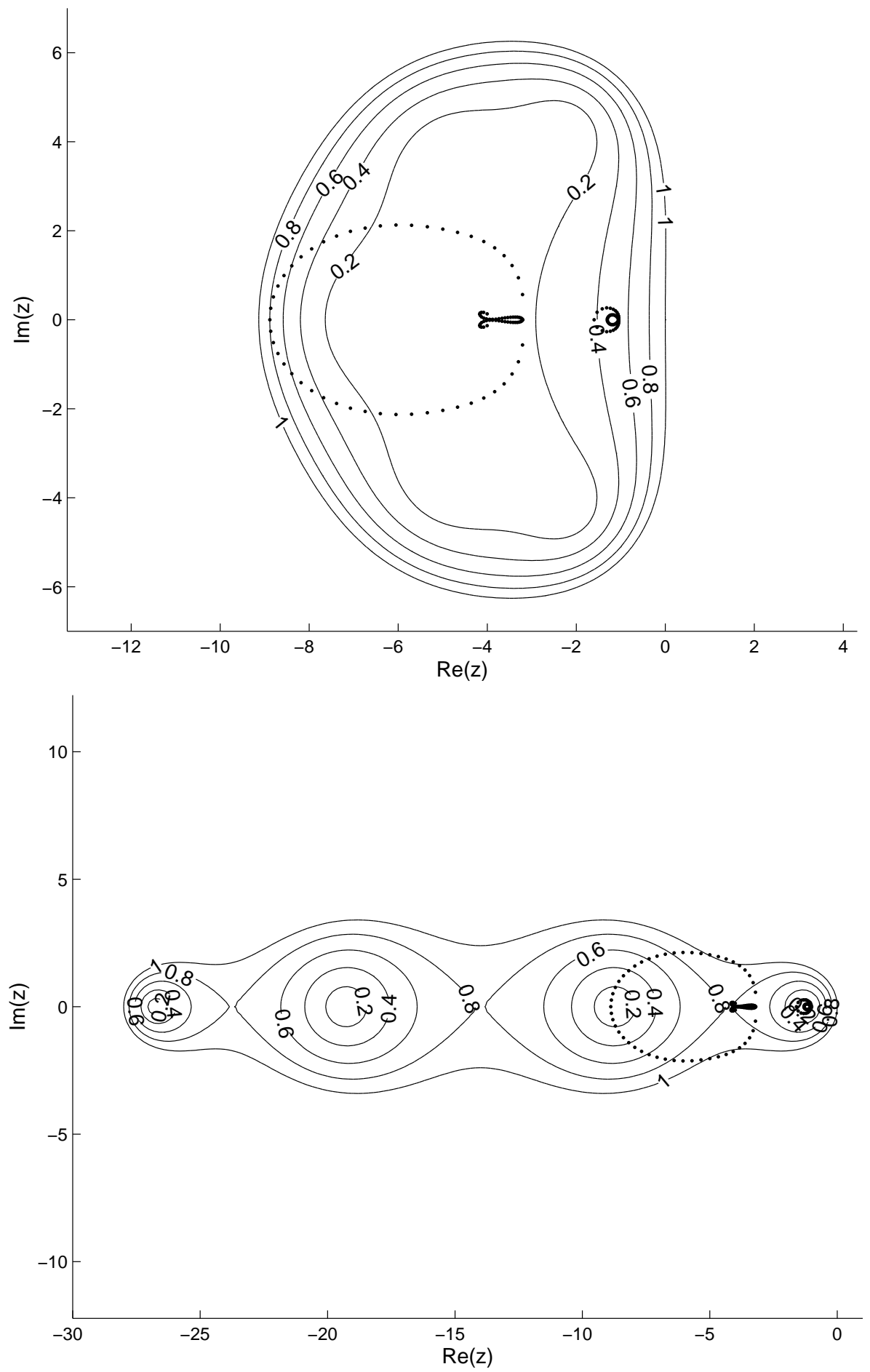

Fig. 3. The stability domain $S$ and values $-\lambda \mu$ (dots) for the EXI method (top) and EXV method (bottom) in the time-dependent inviscid flow regime with $\lambda=1.6$. The pseudo-time CFL number is 1.6 and for this constraint only the EXI method is stable. 

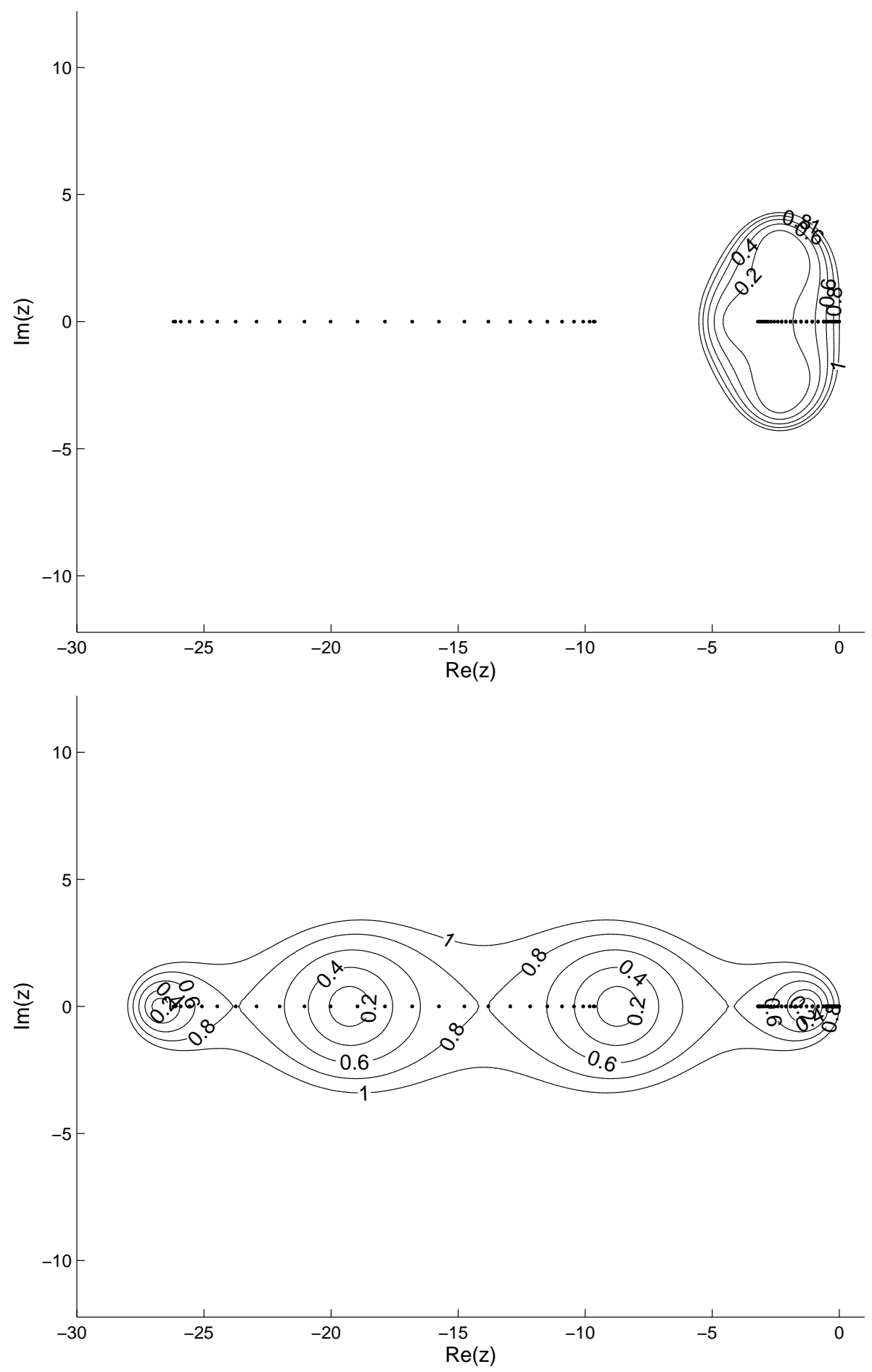

Fig. 4. The stability domain $S$ and values $-\lambda \mu$ (dots) for the EXI method (top) and EXV method (bottom) in the time-dependent viscous flow regime with $\lambda=8 \cdot 10^{-3}$. The pseudo-time Von Neumann number is 0.8 and for this constraint only the EXV method is stable. 


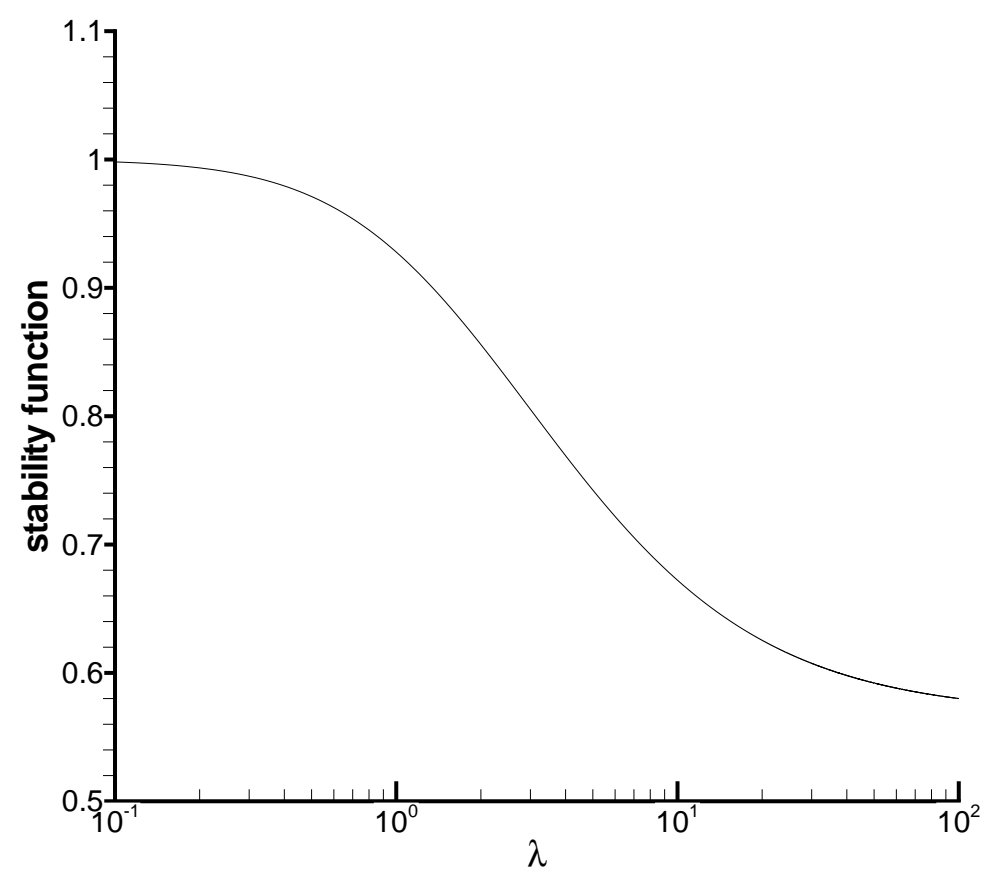

Fig. 5. The stability function $f$ for $\left\|P_{\mathcal{A}}\right\|=1$. 


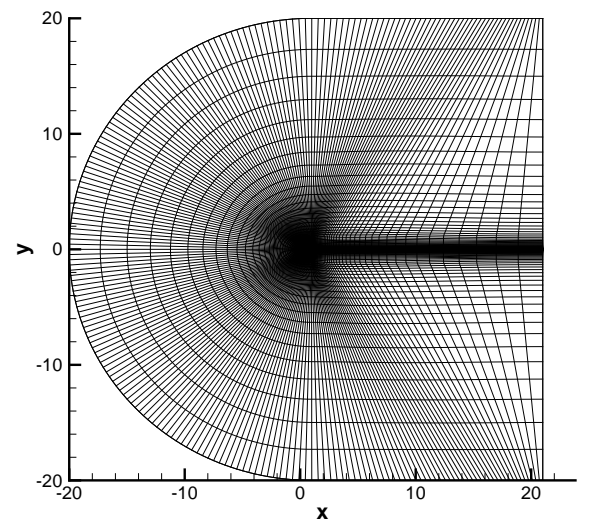

(a) Overview

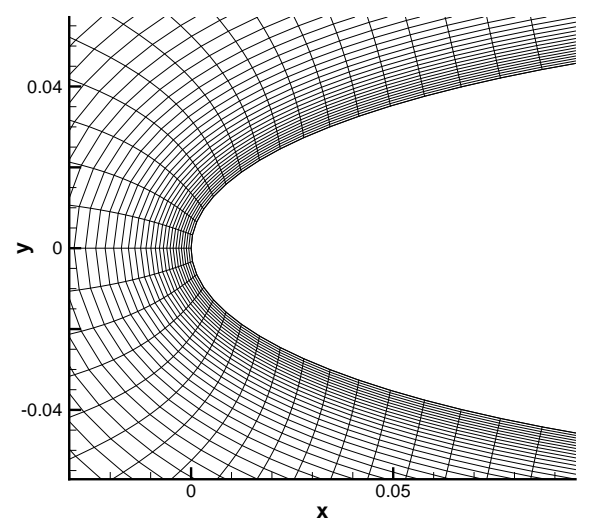

(c) Nose

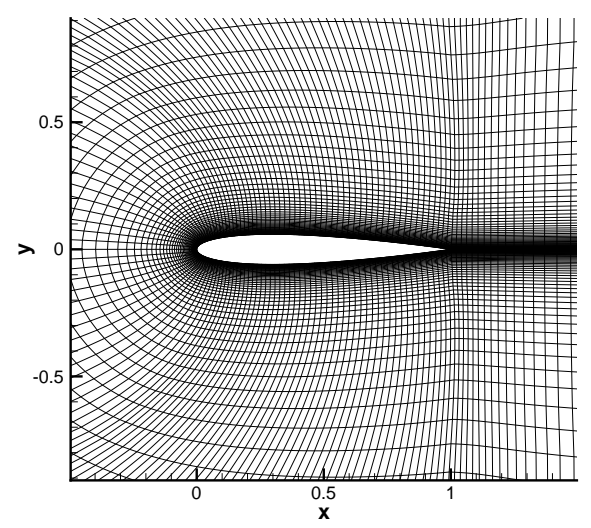

(b) Airfoil

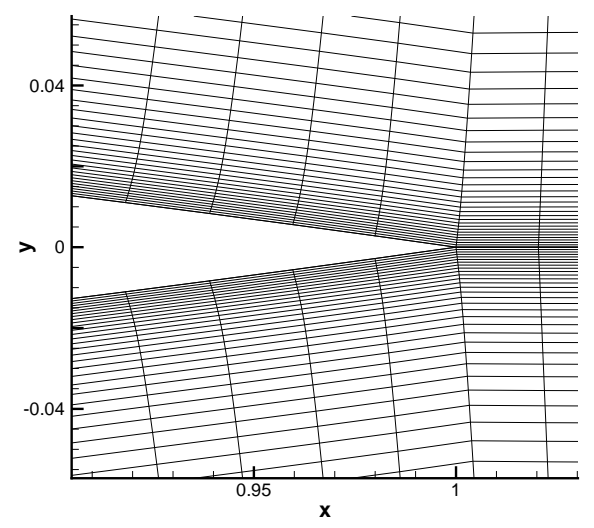

(d) Tail

Fig. 6. Details of the NACA0012 C-grid with $224 \times 76$ elements. 

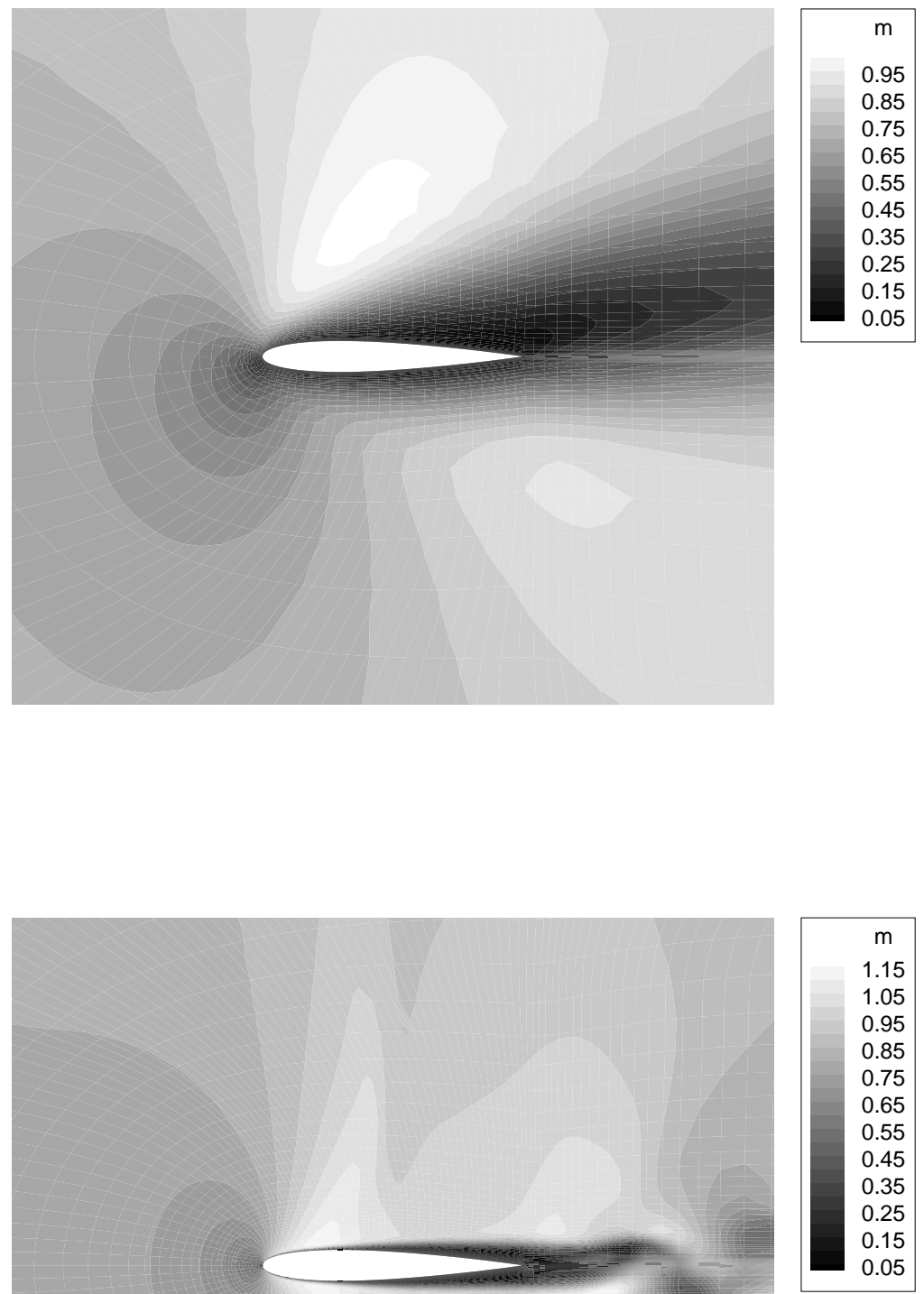

Fig. 7. Local Mach numbers for the $\mathrm{A} 1$ (top; $\mathrm{M}_{\infty}=0.8, \operatorname{Re}_{\infty}=73, \alpha=12^{\circ}$ ) and A7 (bottom; $\mathrm{M}_{\infty}=0.85, \mathrm{Re}_{\infty}=10^{4}, \alpha=0^{\circ}$ ) test cases. In the A7 case, snapshot at $t=10$. 

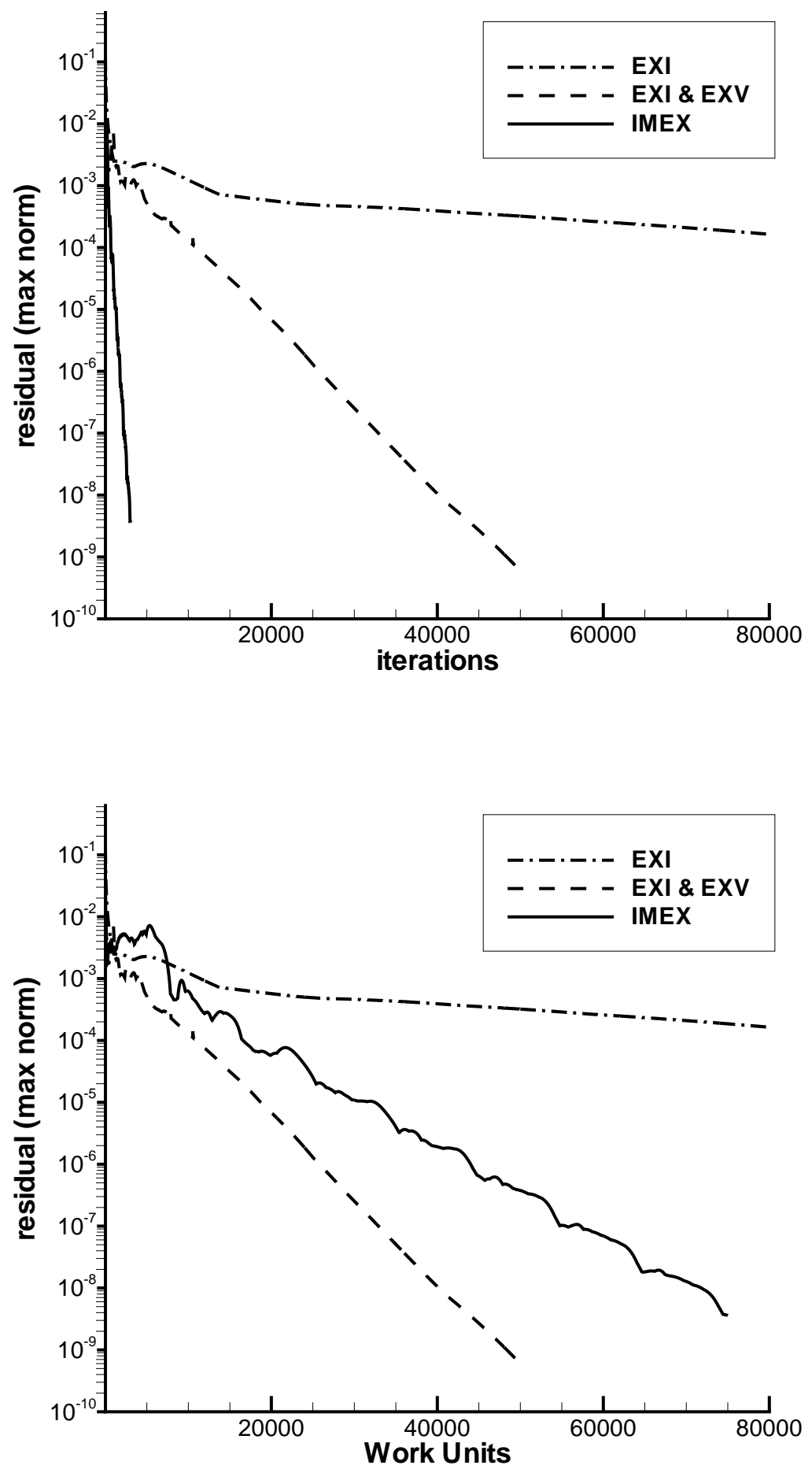

Fig. 8. Convergence to steady state for the $\mathrm{A} 1$ case $\left(\mathrm{M}_{\infty}=0.8, \operatorname{Re}_{\infty}=73, \alpha=12^{\circ}\right)$ on the $112 \times 38$ grid in terms of iterations (top) and work units (bottom). 

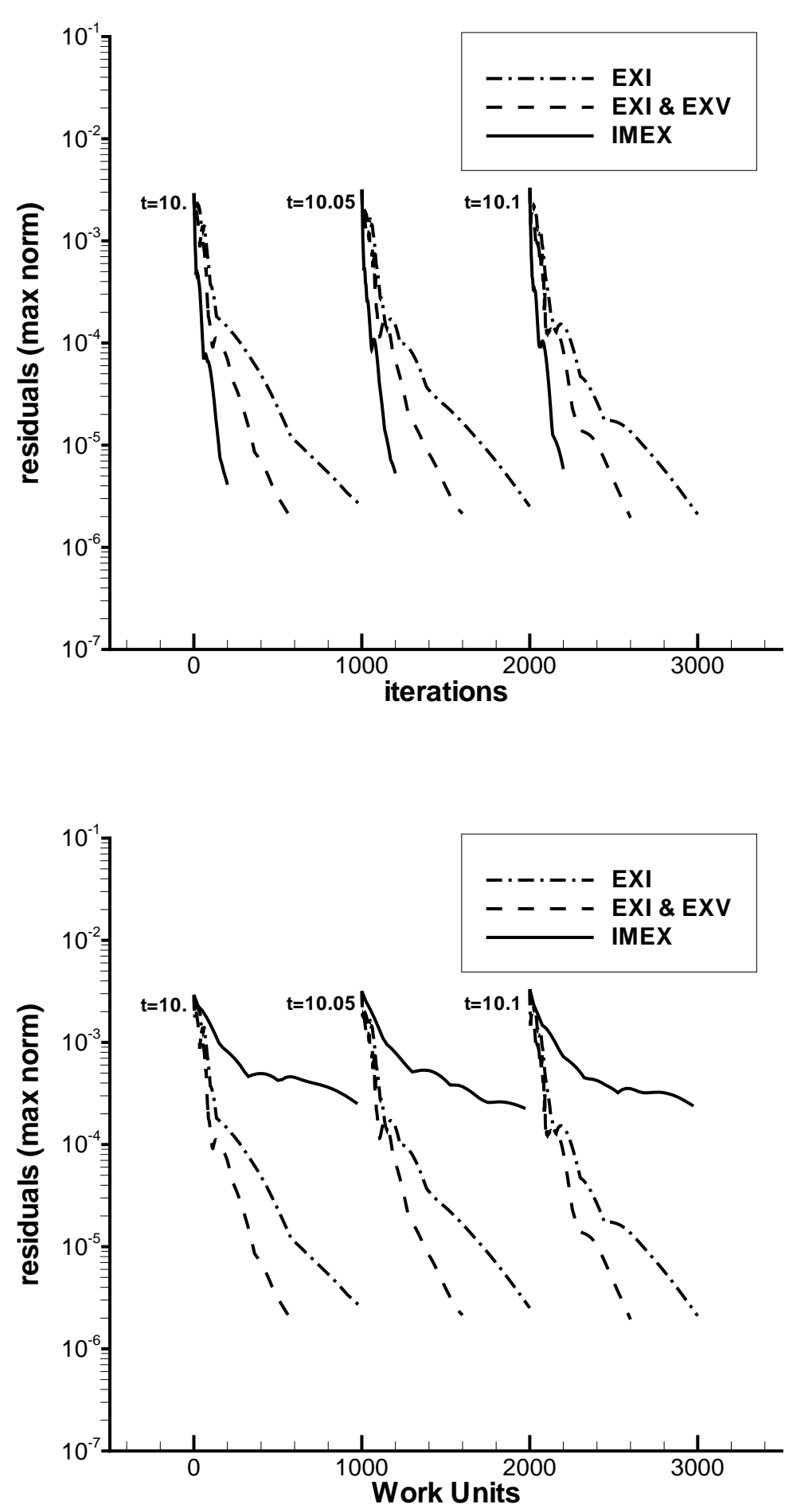

Fig. 9. Convergence in pseudo-time for three physical time steps in the A7 case $\left(\mathrm{M}_{\infty}=0.85, \mathrm{Re}_{\infty}=10^{4}, \alpha=0^{\circ}\right)$ on the $224 \times 76$ grid, expressed in terms of iterations (top) and work units (bottom). 
Table 1

Stability constraints of the EXI and EXV methods.

\begin{tabular}{lcccc}
\hline \hline & \multirow{2}{*}{ flow regime } & \multicolumn{2}{c}{ stability restriction } \\
& $\sigma$ & $\operatorname{Re}_{\Delta x}$ & EXI & EXV \\
\hline Steady-state, inviscid & 100 & 100 & $\sigma_{\Delta \tau} \leq 1.8$ & $\sigma_{\Delta \tau} \leq 0.3$ \\
Steady-state, viscous & 100 & 0.01 & $\delta_{\Delta \tau} \leq 0.1$ & $\delta_{\Delta \tau} \leq 0.8$ \\
Time-dependent, inviscid & 1 & 100 & $\sigma_{\Delta \tau} \leq 1.6$ & $\sigma_{\Delta \tau} \leq 1.0$ \\
Time-dependent, viscous & 1 & 0.01 & $\delta_{\Delta \tau} \leq 0.1$ & $\delta_{\Delta \tau} \leq 0.8$ \\
\hline \hline
\end{tabular}

